\title{
Sulle intersezioni di tre superficie algebriche.
}

\author{
(Di Lurgr Berzolari, a Torino.)
}

Scopo principale di questo lavoro è la dimostrazione (algebrica) rigorosa del seguente teorema fondamentale:

Date tre qualunque superficie algebriche di ordini $l, m, n$, semplicio comunque composte (anche dotate di infiniti punti multipli), ma non passanti per una medesima curva, la condizione necessaria e sufficiente perchè in un loro punto comune 0 , dove esse hanno rispettivamente le multiplicità $\lambda, \mu, \nu$, siano raccolte precisamente $\lambda \mu \nu, e$ non più, delle loro lmn intersezioni, $\grave{e}$ che $i$ tre coni tangenti alle superficie nel punto 0 non abbiano in comune nessuna generatrice, qualunque siano del resto le singolarità presentate da quei coni.

La dimostrazione che ne daro sussiste, senza modificazioni sostanziali, pel caso di $n(>2)$ varietà algebriche (ad $n-1$ dimensioni) dello spazio ad $n$ dimensioni, e soltanto per evitare complicazioni di pura forma viene qui esposta per $n=3$. Essa è un'estensione (che non si presenta però del tutto ovvia) di quella che fu data per la prima volta dal sig. Yoss $\left(^{*}\right)$ per due curve

(*) Voss, Ueber einen Fundamenhalsats aus der Theove der algebraischen Funetionen (Wath. Annalen, Bd. 27, pag. 533 e seg.). - V. altresi l'osservazione del sig. Noether in una noti a piè della pag. 144 del vol. 40 dei Math. Ann.

Nelle sue lezioni del $1894-95$ il prof. SEgre ha approfondito ulteriormente la questione, estendendo il calcolo stesso del sig. Voss al caso in cui le due curve abbiano nel punto che si considera una tangente comune, e cercando allora quante intersezioni delle due curve vengano a cadere in quel punto. Come applicazioni imamediate di questi risultati alle intersezioni di una curva con le prime polari di punti generici o di punti della curva o di una tangente singolare, come pure alle intersezioni della curva con la

Annali di Malemaica, tomo XXIV. 
piane, e che fu poi riportata dal sig. Bertins nella Nota che ha per titolo Rappresentazione di una forma ternaria per combinazione lineare di due altre (Rendic. del $\mathrm{R}$. Istituto Lombardo, serie 2. ${ }^{\mathrm{a}}$, vol. 24, 5 novembre 1891).

In seguito, supponendo che $\mathrm{i}$ tre coni sopra nominati contengano una stessa generatrice, la quale abbia inoltre riunite in 0 quante si vogliano intersezioni con ciascuna delle date superficie, determino il numero delle intersezioni di queste ultime che vengono ulteriormente a cadere in generale nel punto 0 , assegnando tutti $\mathrm{i}$ casi in cui questo numero diventa ancor più elevato. Per ultimo, come applicazione dei risultati ottenuti, dimostro alcune proposizioni relative all'abbassamento che un punto multiplo di una superficie produce nella classe di questa, considerando in modo particolare il caso in cui il cono tangente nel punto multiplo possiede una generatrice doppia.

Una dimostrazione rigorosa del teorema enunciato in principio, per quanto mi consta, non è stata data finora, neanche per lo spazio ordinario, dove di solito la proprietà viene ammessa come intuitiva; $i$ risultati rínanenti $\left(n .{ }^{i} 7\right.$, 8,9 e 10) sono del tutto nuovi.

Sembrami anche degno di nota, per la sua eleganza, il molo col quale si prova nel $\mathrm{n}^{\circ} 2$ che le tre superficie hanno $\operatorname{lm} n$ punti comuni, benchè di questo, come di altri analoghi teoremi, si conoscano già (anche per un iperspazio) parecchie dimostrazioni.

1. In coordinate omogenee siano

$$
\left.\begin{array}{l}
A \equiv a_{00}+\left(a_{10} x_{1}+a_{14} x_{2}\right)+\cdots+\left(a_{10} x_{1}{ }^{l}+\cdots+a_{l l} x_{2}{ }^{b}\right)=0, \\
B \equiv b_{00}+\left(b_{10} x_{1}+b_{11} x_{2}\right)+\cdots+\left(b_{m 0} x_{1}^{m}+\cdots+b_{m m} x_{2}{ }^{m}\right)=0, \\
C \equiv c_{00}+\left(c_{10} x_{1}+c_{11} x_{2}\right)+\cdots+\left(c_{n 0} x_{1}^{n}+\cdots+c_{n n} x_{2}{ }^{n}\right)=0
\end{array}\right\}
$$

le equazioni delle tre superficie $A, B, C$, dove per es. le $a_{i n}, a_{i}, \ldots, a_{i i}$ sono forme binarie di grado $l-i$ nelle $x_{3}, x_{1}$. Volendo eliminare dalle (1) le $x_{1}$, $x_{2}$, non si conosce finora (a meno che $l, m, n$ non siano fra loro uguali) un'espressione della risultante, che, essendo priva di fattori estranei, abbia

llessiana, egli ha poi stabilite le proposizioni fondamentali sulle curve piane algebriche a sulle loro singolarita piu comuni, con precisione e rigore magziori di quanto non si trovi nei noti trattati classici. Ad es. egli dimostro per questa via le proposizioni analoghe a quelle che per le superficie io trattero alla fine del presente lavoro $\left(n^{\circ} 10\right)$. 
la forma di determinante. È perd dovuto al $\mathrm{C}_{\triangle \mathrm{XLEY}}\left(^{*}\right)$ un metodo per ottenere quella risultante come quoziente di due determinanti; ed ecco, brevemente, in che consiste. Moltiplichiamo le (1) risp. per

$$
\begin{aligned}
& 1 ; x_{1}, x_{2} ; x_{1}^{2}, x_{1} x_{2}, x_{2}^{2} ; \ldots ; x_{1}^{m+n-2}, x_{1}^{m+n-3} x_{2}, \ldots, x_{8}^{m+n-2} ; \\
& 1 ; x_{1}, x_{2} ; \ldots \ldots \ldots \ldots ; x_{1}^{n+l-2}, x_{1}^{n+l-3} x_{2}, \ldots, x_{2}^{n+l-2} \\
& 1 ; x_{1}, x_{2} ; \ldots \ldots \ldots ; x_{1}^{l+m-2}, x_{1}^{l+m-3} x_{2}, \ldots, x_{2}^{l+m-2} \text {. }
\end{aligned}
$$

Otteniamo cos̀̀

$$
\frac{1}{2}(m+n)(m+n-1)+\frac{1}{2}(n+l)(n+l-1)+\frac{1}{2}(l+m)(l+m-1)
$$

equazioni lineari fra le quantità

$$
1 ; x_{1}, x_{2} ; \ldots ; x_{1}^{l+m+n-2}, x_{1}^{l+m+n-3} x_{2}, \ldots, x_{2}^{l+m+n-\varepsilon},
$$

che sono in numero di

$$
\frac{1}{2}(l+m+n)(l+m+n-1) .
$$

Tali equazioni non sono però fra loro indipendenti: invero, se si moltiplicano le identità

$$
B C=C B, \quad C A=A C, \quad A B=B A
$$

risp. per

$$
\begin{aligned}
& 1 ; \quad x_{1}, x_{2} ; \ldots ; \quad x_{1}^{l-2}, x_{1}^{l-3} x_{2}, \ldots, x_{2}^{l-8} \\
& 1 ; x_{1}, x_{2} ; \ldots ; x_{1}^{m-2}, x_{1}^{m-3} x_{2}, \ldots, x_{2}^{m-2} ; \\
& 1 ; x_{1}, x_{2} ; \ldots ; x_{1}^{n-8}, x_{1}^{n-s} x_{2}, \ldots, x_{2}^{n-2} \text {, }
\end{aligned}
$$

si ottengono

$$
\frac{1}{2} l(l-1)+\frac{1}{2} m(m-1)+\frac{1}{2} n(n-1)
$$

relazioni lineari fra le precedenti equazioni: il numero (4) è la differenza fra

(*) CAYLEY, On the Theory of Elimination (Cambridge and Dublin Math. Journal, vol. III, pag. 116; oppure The Collected Mathem. Papers, vol, I, pag. 370;. - La teoria di GAYLEY è riprodotta succintamente nell'Algebra superiore di SALMox (pag. 125 e seg. della 2.a ediz. francese). 
i numeri (2) e (3). Ad. es., la prima di tali relazioni è:

$$
\begin{aligned}
c_{00} \cdot B+ & \left(c_{10} \cdot x_{1} B+c_{11} \cdot x_{2} B\right)+\cdots+ \\
& +\left(c_{n 0} \cdot x_{1}^{n} B+c_{n 1} \cdot x_{1}^{n-1} x_{2} B+\cdots+c_{n n} \cdot x_{2}^{n} B\right) \\
-b_{00} \cdot C- & \left(b_{10} \cdot x_{1} C+b_{11} \cdot x_{2} C\right)-\cdots- \\
& -\left(b_{m 0} \cdot x_{1}{ }^{m} C+b_{m 1} \cdot x_{1}^{m-1} x_{2} C+\cdots+b_{m i n} \cdot x_{2}{ }^{n} C\right)=0 .
\end{aligned}
$$

Si formino ora due matrici $H$ ed $H$, le quali abbiano risp. per elementi i coefficienti delle equazioni sopra costruite, e quelli delle relazioni ultimamente trovate. Entrambe conterranno un numero di linee dato dall'espressione (2), mentre il numero delle loro colonne sarà dato risp. dalle (3) e (4). Allora il CAyLex ha trovato che la risultante $R$ delle (1) si può ottenere dividendo uno qualunque dei determinanti tratti dalla matrice $H$ (il quale non sia nullo identicamente) per il determinante supplementare tratto dalla matrice $H^{\prime}$, ciò̀ per quello formato con le linee di $H^{\prime}$ aventi lo stesso posto di quelle che si sono dianzi trascurate in $H$. Di guisa che, indicando con $\chi^{\mathrm{e}}$ $\chi^{\prime}$ due tali determinanti, si avrà:

$$
R=\frac{\chi}{\psi^{\prime}}
$$

Il modo di composizione delle matrici $H$ ed $H^{\prime}$ risulta del tutto chiaro dalle osservazioni seguenti, nelle quali introduciamo altresi alcune notazioni, che si manteranno in tutto il lavoro. La $H$ è costituita da tre matrici parziali, che diremo $1,2,3$, di cui tutti gli elementi appartengono come coefficienti soltanto alla prima, od alla seconda, od alla terza delle (1). La 1 contiene $m+n-1$ gruppi di linee, formati risp. di $1,2, \ldots, m+n-1$ linee; la 2 ne contiene $n+l-1$, formati risp. di $1,2, \ldots, n+l-1$ linee; la 3 ne contiene $l+m-1$, formati risp. di $1,2, \ldots, l+m-1$ linee. Le colonne di $H$ si dividono invece in $l+m+n-1$ gruppi, contenenti ordinatamente $1,2, \ldots, l+m+n-1$ colonne. Mediante siffatti gruppi di linee e colonne la matrice 1 risulta divisa in $(l+m+n-1)(m+n-1)$ matrici parziali; e chiamando $\alpha_{i j}$ quella che è comune all' $i^{\text {mo }}$ gruppo di linee ed all' $j^{m o}$ gruppo di colonne, si ha:

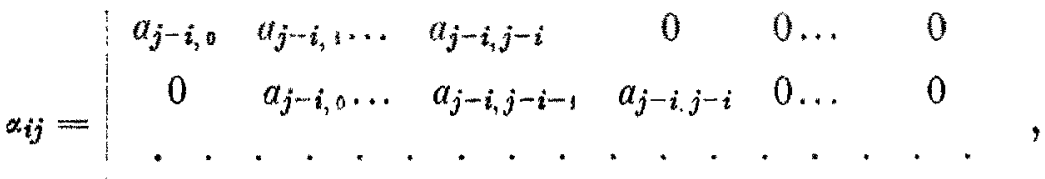

$$
\begin{aligned}
& \begin{array}{lllllll}
0 & 0 & . & . & . & . & . \\
a_{j-i j-i}
\end{array}
\end{aligned}
$$


dove la matrice del secondo membro contiene $i$ linee e $j$ colonne: ogni suo elemento che non sia nullo è una forma binaria di grado $l-j+i$ in $x_{3}, x_{1}$. Denotando analogamente con $\beta_{i j}, \gamma_{i j}$ le matrici comuni all' $i^{\text {mo }}$ gruppo di linee ed all' $j^{\text {mo }}$ gruppo di colonne di 2 e 3 , e scrivendo 0 per significare una matrice, di cui tutti gli elementi sono nulli, la matrice $H$ si può scrivere come segue:

$$
\begin{aligned}
& \begin{array}{lllllll}
\alpha_{11} & \alpha_{12} & \alpha_{13} \ldots & \alpha_{1, l+1} & 0 & 0 \ldots & 0
\end{array} \\
& \begin{array}{lllllll}
0 & \alpha_{22} & \alpha_{23} \ldots & \alpha_{3, l+:} & \alpha_{3, l+2} & 0 \ldots & 0
\end{array} \\
& \begin{array}{llllllllll}
0 & 0 & 0 & . & . & . & . & . & \alpha_{m+n-1, l+m+n-1}
\end{array}
\end{aligned}
$$

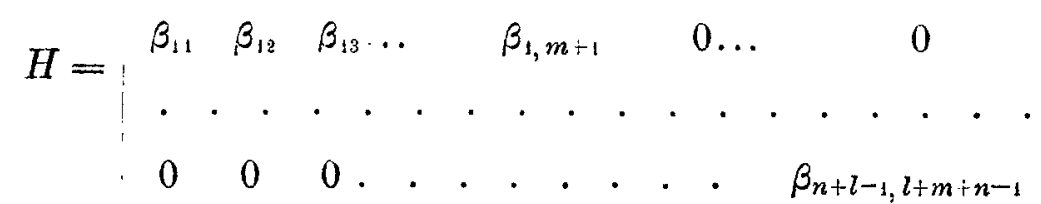

$$
\begin{aligned}
& \begin{array}{llllll}
\gamma_{11} & \gamma_{12} & \gamma_{13} \ldots & \gamma_{1, n+1} & 0 \ldots & 0
\end{array} \\
& \begin{array}{lllllllllllll}
0 & 0 & 0 & . & . & . & . & . & . & \gamma l+m-1, l+m+n-1
\end{array}
\end{aligned}
$$

La matrice $H^{\prime}$ è formata di tre matrici parziali $1^{\prime}, 2^{\prime}, 3$, di cui ciascuna, per ciò che riguarda il numero e la disposizione dei gruppi di lince, nonchè delle linee di ciascun gruppo, è formata nell'jdentico modo delle matrici 1, 2, 3 di $H$. Quanto alle colonne, la $H^{\prime}$ si scinde in tre matriei $\mathrm{I}^{\prime}$, II', III' : la $\mathrm{I}^{\prime}$ contiene $n-1$ gruppi, cui appartengono ordinatamente 1 , $2, \ldots, n-1$ colonne; la $\mathrm{II}^{\prime}$ ne contiene $l-1$ con $1,2, \ldots, l-1$ coloune risp., e la III' ne contiene $m-1$ con risp. $1,2, \ldots, m-1$ colonne. Mediante siffatte matrici di linee e di colonne, la $H^{\prime}$ risulta scomposta (oltre che in tre di elementi tutti nulli) in sei nuove matrici, ciascuna delle quali è formata coi coefficienti di una sola delle (1). Ognuna alla sua volta risulta poi composta di matrici minori, tali che tutti gli elementi di una qualunque di esse sono di uno stesso grado in $x_{3}, x_{1}$ : se una di tali matrici minori è quella comune all' $i^{m o}$ gruppo di linee ed all' jmo gruppo di colonne, per es., della matrice $2^{\prime} I^{\prime}$, ogni suo elemento è una forma di grado $l-i+j$ in $x_{3}, x_{4}$. Sono nulli tutti gli elementi delle tre matrici $1^{\prime} \mathrm{II}^{\prime}, 2^{\prime} \mathrm{III}^{\prime}, 3^{\prime} \mathrm{I}^{\prime}$; mentre gli elementi delle matrici $1^{\prime} I^{\prime}, 2^{\prime}$ II , $3^{\prime}$ III' si trovano tutti, rispetto. 
alle (1), cambiati di segno. Possiamo dunque scrivere:

$$
\begin{aligned}
& \text { I } \text { II' III }^{\prime} \\
& \begin{array}{lllllllllllll}
-b_{\mathrm{00}} & 0 & 0 \ldots & 0 & 0 \ldots & 0 & \mid & \mid c_{00} \ldots & 0 & 0 \ldots & 0
\end{array} \\
& \begin{array}{llllllllllll}
-b_{10}-b_{10} & 0 \ldots & 0 & 0 \ldots & 0 & \mid & \mid c_{80} \ldots & 0 & 0 \ldots & 0
\end{array} \\
& \begin{array}{llllllllllll}
-b_{11} & 0 & -b_{00} \ldots & 0 & 0 \ldots & 0 & 1 & \mid c_{11} \ldots & 0 & 0 \ldots & 0
\end{array} \\
& \text { o } \\
& \begin{array}{lllllllllll}
0 & 0 & 0 \ldots & -b_{m 1}-b_{m 0} \ldots & 0 & 1 & 0 \ldots & c_{n 1} & c_{n 0} \ldots & 0
\end{array} \\
& \ldots \ldots \ldots \ldots \ldots \\
& \begin{array}{llllll:lllll}
0 & 0 & 0 \ldots & 0 & 0 \ldots & -b_{n 0} \mid & 0 \ldots & 0 & 0 \ldots & c_{n 0}
\end{array} \\
& \ldots-\cdots-\cdots-1-\cdots-\cdots \\
& H=\begin{array}{cccccc:cccc}
a_{00} & 0 & 0 \ldots & 0 & 0 \ldots & 0 & -c_{00} \ldots & 0 & 0 \ldots & 0 \\
\ldots & \ldots & \ldots & \ldots & \ldots & \ldots & \ldots & \ldots & \ldots & \ldots
\end{array} \\
& \begin{array}{|cccccc:cccc}
0 & 0 & 0 \ldots & a_{l_{0}} & 0 \ldots & 0 & 0 \ldots & -c_{n 0} & 0 \ldots & 0 \\
\ldots & \ldots & \ldots & \ldots & \ldots & \ldots & \ldots & \ldots & \ldots & \ldots
\end{array}
\end{aligned}
$$

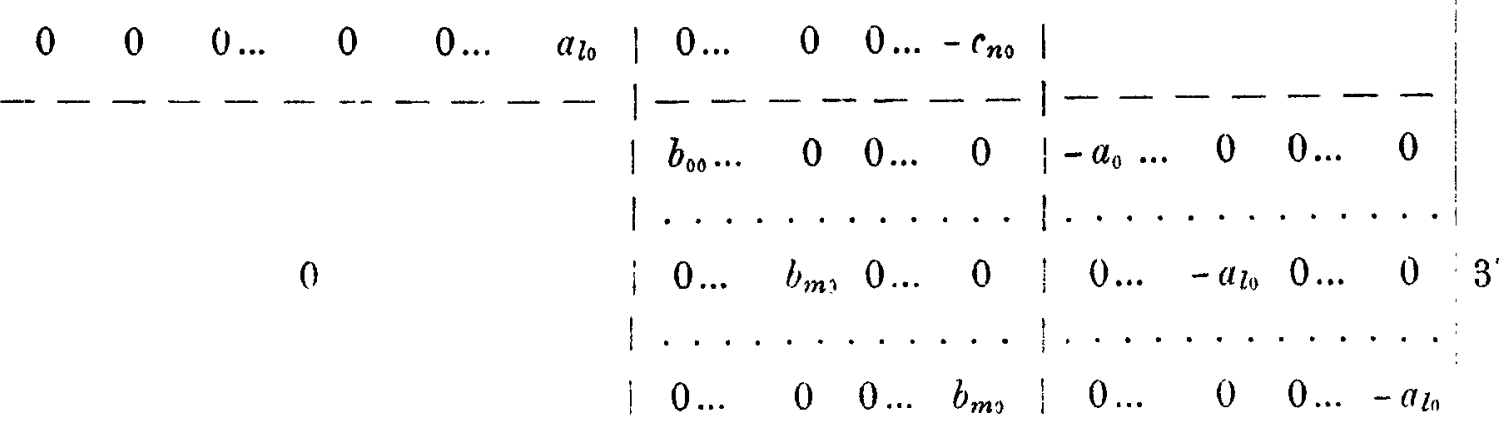

2. Clò posto, poichè per ipotesi il numero delle intersezioni delle tre date superficie d̀ finito, possiamo supporre di aver scelto il tetraedro di riferimento in modo che il suo spigolo $x_{3}=x_{4}=0$ non sia tagliato da nessuna delle congiungenti due di quelle intersezioni. L'equazione

$$
R=0 \text {, }
$$

che rappresenta l'insieme dei piani projettanti dal detto spigolo quelle intersezioni, avrà quindi tante radiei $\frac{x_{3}}{x_{4}}$ quante sono le intersezioni stesse, sicchè i) numero di queste ultime si avrà cercando il grado di $R$, cioè la differenza dei gradi di $x$ e $\chi^{\prime}$. 
Per trarre da $H$ il determinante $\chi$, prendiamo in generale $\rho_{i}$ linee del gruppo $i^{\text {mo }}$ della matrice $1(i=1,2, \ldots, m+n-1), \sigma_{i}$ linee del gruppo $i^{\text {mo }}$ della matrice $2(i=1,2, \ldots, n+l-1)$, e $\tau_{i}$ linee del gruppo $i^{m o}$ della matrice $3(i=1,2, \ldots, l+m-1)$. Analogamente, per trarre da $H^{\prime}$ il determinante $\chi^{\prime}$, prendiamo $\rho_{i}^{\prime}$ linee del gruppo $i^{m o}$ della matrice $1^{\prime}(i=1$, $2, \ldots, m+n-1)$, e cosi via.

Poichè gli ordini di $\chi$ e $\chi$ sono dati risp. dalle espressioni (3) e (4), si avrà :

$$
\begin{aligned}
& \sum_{1}^{m+n-1} \rho_{i}+\sum_{1}^{n+l-1} \sigma_{i}+\sum_{1}^{l+m-1} \tau_{i}=\frac{(l+m+n)(l+m+n-1)}{2}, \\
& \sum_{1}^{m+n-1} \rho_{i}+\sum_{1}^{n+l-1} \sigma_{i}^{\prime}+\sum_{i}^{l+m-1} \tau_{i}^{\prime}=\frac{l(l-1)+m(m-1)+n(n-1)}{2} .
\end{aligned}
$$

Inoltre sarà

$$
\rho_{i}+\rho_{i}^{\prime}=i, \quad \sigma_{i}+\sigma_{i}^{\prime}=i, \quad \tau_{i}+\tau_{i}^{\prime}=i
$$

per tutti $i$ valori di $i$ che ranno da 1 risp. fino ad $m+n-1, n+l-1$, $l+m-1$.

Un elemento qualsiasi di $\chi$ si otterrà scegliendo ordinatamente in ciascuna delle $\rho_{1}$ linee del primo gruppo della matrice 1 un elemento, la cui colonna corrispondente apparterrà ad un gruppo di posto $r_{11}, r_{12}, \ldots, r_{1 p_{1}}$; indi in ciascuna delle $p_{2}$ linee del secondo gruppo di 1 un elemento situato in una colonna appartenente ad un gruppo di posto $r_{21}, r_{22}, \ldots, r_{2 p_{z}} ; \ldots ;$ in ciaseuna delle $\rho_{m+n-1}$ linee dell' ultimo gruppo di 1 un elemento situato in una colonna di un gruppo di posto $r_{m+n-1,1}, \ldots, r_{m+n-1, \rho_{m \rightarrow n-1}}$; e facendo l'operazione analoga per le diverse linee fissate nei vari gruppi delle matrici 2 e 3 , col che si verranno ad introdurre, con significato analogo a quello dei numeri $r$, nuovi numeri che chiameremo $s_{11}, s_{12}, \ldots, s_{1 s_{1}} ; s_{21}, \ldots, s_{2 \sigma_{2}} ; \ldots$; $s_{n+l-1,1}, \ldots, s_{n+l-1, \sigma_{n+2-1}} ;-t_{11}, t_{12}, \ldots, t_{1 \tau_{1}} ; t_{21}, \ldots, t_{2 \tau} ; \ldots ; t_{l+m-1,1}, \ldots$, $t_{l+m-1, i+m-1}$. In tal guisa rengono a considerarsi, prescindendo dall' ordine, tutte le colonne della matrice $H$, onde:

ossia:

$$
\mathbf{\Sigma} r+\mathbf{\Sigma} s+\mathbf{\Sigma} t=1^{2}+2^{2}+\cdots+(l+m+n-1)^{2}
$$

$$
\mathbf{Z} r+\mathbf{\Sigma} s+\mathbf{\Sigma} t=\frac{(l+m+n)(l+m+n-1)(2 l+2 m+2 n-1)}{6} .
$$

Un elemento qualunque del determinante $\chi$ si formerz̀ in modo analogo al precedente, e s'introdurranno cosi dei numeri $r_{11}^{\prime}, \ldots, r^{\prime}{ }_{1 p_{1}^{\prime}}^{\prime} ; \ldots ; r_{m+n-\}_{1}, 1}^{\prime}, \ldots$, 
$r_{m+n-1, \rho^{\prime}}^{\prime} ; s_{11+n-1}^{\prime}, \ldots, s_{n+l-1, \sigma^{\prime}}^{\prime}, t_{n+l-1}^{\prime}, \ldots, t_{l+m-1, \tau^{\prime}+m-1}^{\prime}$, legati dalla relazione

$$
\begin{gathered}
\mathbf{\Sigma} \boldsymbol{r}^{\prime}+\mathbf{\Sigma} s^{\prime}+\mathbf{\Sigma} t^{\prime} \\
=l(l-1)(2 l-1)+m(m-1)(2 m-1)+n(n-1)(2 n-1) \\
\end{gathered}
$$

Pertanto il grado di $\chi$ in $x_{3}, x_{4}$ è

$$
\begin{aligned}
& \sum_{i=1}^{i=o_{1}}\left(l-r_{1 i}+1\right)+\sum_{i=1}^{i=i^{2}}\left(l-r_{2 i}+2\right)+\cdots+\sum_{i=1}^{i=0}\left(l-r_{m+n-1, i}+m+n-1\right) \\
+ & \sum_{i=1}^{i=s_{1}}\left(m-s_{i i}+1\right)+\sum_{i=1}^{i=i^{2}}\left(m-s_{2 i}+2\right)+\cdots+\sum_{i=1}^{i=\sum^{n+l-1}}\left(m-s_{n+l-1, i}+n+l-1\right) \\
+ & \sum_{i=1}^{i=\tau_{1}}\left(n-t_{1 i}+1\right)+\sum_{i=1}^{i=\tau_{2}}\left(n-t_{2 i}+2\right)+\cdots+\sum_{i=1}^{i=i_{l+1}^{m-1}}\left(n-t_{l+m-1, i}+l+m-1\right),
\end{aligned}
$$

ossia

$$
\sum_{i=1}^{i=m+n-1}(l+i) \rho_{i}+\sum_{i=1}^{i=n+l-1}(m+i) \sigma_{i}+\sum_{i=1}^{i=l+m-1}(n+i) \tau_{i}-(\mathbf{\Sigma} r+\mathbf{\Sigma} s+\mathbf{\Sigma} t)
$$

Quanto al grado di $\chi^{\prime}$, si osservi che i numeri $r^{\prime}, s^{\prime}, t^{\prime}$ denotano il posto delle colonne scelte, quando si considerino separatamente le tre matrici I', II', III', sicchè ad es. un $r^{\prime}$ può riferirsi all' $r^{\prime}$ mo gruppo tanto di I' quanto di III'. Se quindi diciamo $\approx$ un numero che può coincidere tanto con $m$ quanto con $n$, $y$ un numero che può coincidere tanto con $n$ quanto con 7, e $z$ un numero che può essere tanto $l$ quanto $m$, il grado di $\chi^{\prime}$ sarà

$$
\begin{aligned}
& \sum_{i=1}^{i=y^{\prime}}\left(x+r^{\prime}{ }_{1 i}-1\right)+\sum_{i=1}^{i=P^{\prime}}\left(x+r^{\prime}{ }_{2 i}-2\right)+\cdots+\sum_{i=1}^{i=p^{\prime}}\left(x+n-r_{m+n-1, i}-m-n+1\right)
\end{aligned}
$$

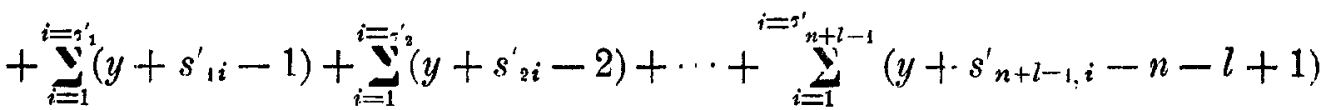

$$
\begin{aligned}
& +\sum_{i=1}^{i=\tau^{\prime}}\left(z+t_{1 i}-1\right)+\sum_{i=1}^{i=\tau^{\prime}}\left(z+t_{2 i}^{\prime}-2\right)+\cdots+\sum_{i=1}^{i=\tau^{\prime}+m-1}\left(z+t_{l+m-1, i}-l-m+1\right),
\end{aligned}
$$

ossia

$$
\mathbf{\Sigma} x+\mathbf{\Sigma} y+\mathbf{\Sigma} z+\mathbf{\Sigma} r^{\prime}+\mathbf{\Sigma} s^{\prime}+\mathbf{\Sigma} t^{\prime}-\sum_{i=1}^{i=m+n-1} i \rho_{i}^{\prime}-\sum_{i=1}^{i=n+l-1} i \sigma_{i}-\sum_{i=1}^{i=l+m-1} i \tau^{\prime}{ }_{i}
$$


Ora, per quanto concerne $\mathbf{\Sigma} x$, nel considerare le $\sum_{i}^{m+n-1} \rho_{i}^{\prime}$ colonne avremo preso un certo numero $b^{\prime}$ di volte un elemento $b$ ed un certo numero $c^{\prime}$ di volte un elemento $c$, onde sarà:

$$
\begin{aligned}
& b^{\prime}+c^{\prime}=\sum_{1}^{m+n-1} \rho^{\prime}, \\
& \mathbf{\Sigma} x=m b^{\prime}+n c^{\prime} .
\end{aligned}
$$

Così pure, nel considerare le $\sum_{1}^{n+7-1} \sigma_{i}^{\prime}$ e poi le $\sum_{1}^{l+m-1} \tau_{i}^{\prime}$ colonne, avremo preso $c^{\prime \prime}$ volte un elemento $c$ ed $a^{\prime \prime}$ volte un elemento $a$, e risp. $a^{\prime \prime}$ volte un elemento $a$ e $b^{\prime \prime \prime}$ volte un elemento $b$. Quindi:

E poi evidente che:

$$
\begin{aligned}
& c^{\prime \prime}+a^{\prime \prime}=\sum_{1}^{n+l-1} \sigma_{i}^{\prime} \\
& \mathbf{y} y=n c^{\prime \prime}+l a^{\prime \prime} \\
& a^{\prime \prime \prime}+b^{\prime \prime}=\underbrace{l+m-1} \tau_{i}^{\prime} \\
& \mathbf{\Sigma} z=l a^{\prime \prime}+m b^{\prime \prime \prime} .
\end{aligned}
$$

$$
b^{\prime \prime \prime}+c^{\prime \prime}=\frac{l(l-1)}{2}, \quad c^{\prime}+a^{\prime \prime}=\frac{m(m-1)}{2}, \quad a^{\prime \prime}+b^{\prime}=\frac{n(n-1)}{2} .
$$

Dalle $\left(9^{\prime}\right),\left(10^{\prime}\right),\left(11^{\prime}\right)$ si deduce:

$$
\mathbf{z}+\mathbf{z} y+\mathbf{z}=l\left(a^{\prime \prime}+a^{\prime \prime}\right)+m\left(b^{\prime \prime}+b^{\prime}\right)+n\left(c^{\prime}+c^{\prime \prime}\right)
$$

ma sommando le $(9),(10),(11)$ a due a due, e tenendo conto delle (12), si ha:

$$
\begin{aligned}
& a^{\prime \prime}+a^{\prime \prime}=\mathbf{\Sigma} a^{\prime}+\mathbf{\Sigma} \tau^{\prime}-\frac{l(l-1)}{2}, \\
& b^{\prime \prime \prime}+b^{\prime}=\mathbf{\Sigma} \tau^{\prime}+\mathbf{\Sigma} \rho^{\prime}-\frac{m(m-1)}{2}, \\
& c^{\prime}+c^{\prime \prime}=\mathbf{\Sigma} \rho^{\prime}+\mathbf{\Sigma} \sigma^{\prime}-\frac{n(n-1)}{2},
\end{aligned}
$$

eppero:

$$
\begin{gathered}
\mathbf{\Sigma} x+\mathbf{\Sigma} y+\mathbf{\Sigma} z=l\left(\mathbf{\Sigma} \sigma^{\prime}+\mathbf{\Sigma} \tau^{\prime}\right)+m\left(\mathbf{\Sigma} \tau^{\prime}+\mathbf{\Sigma} \rho^{\prime}\right)+n\left(\mathbf{\Sigma} \rho^{\prime}+\mathbf{\Sigma} \sigma^{\prime}\right) \\
-\frac{l^{z}(l-1)+m^{2}(n-1)+n^{2}(n-1)}{2} .
\end{gathered}
$$

Annali di Matematica, tomo XxIV. 
Il grado di $\chi^{\prime}$ è quindi

$$
\begin{gathered}
\sum_{i=1}^{i=m+n-1}(n+n-i) \rho_{i}^{\prime}+\sum_{i=1}^{i=n+1-1}(n+l-i) \sigma^{\prime} i+\sum_{i=1}^{i=l+m-1}(l+m-i){r^{\prime}}_{i} \\
+\mathbf{\Sigma} r^{\prime}+\mathbf{\Sigma} s^{\prime}+\mathbf{\Sigma} t^{\prime}-\frac{l^{2}(l-1)+m^{2}(m-1)+n^{2}(n-1)}{2} .
\end{gathered}
$$

Sottraendo quest'espressione dalla (8), si ottiene come grado della risultante $R$ :

$$
\begin{aligned}
& l \mathbf{\Sigma}_{\rho}+m \mathbf{\Sigma} \sigma+n \mathbf{\Sigma} \tau-(m+n) \mathbf{\Sigma}_{\rho^{\prime}}-(n+l) \mathbf{\Sigma} \sigma^{\prime}-(l+m) \mathbf{\Sigma}_{\tau^{\prime}} \\
& +\sum_{i=1}^{i=m+n-1} i\left(\rho_{i}+\rho_{i}^{\prime}\right)+\sum_{i=1}^{i=n+l-1} i\left(\sigma_{i}+\sigma_{i}^{\prime}\right)+\sum_{i=1}^{i=l+m-1} i\left(\xi_{i}+\tau^{\prime}\right) \\
& -\left(\mathbf{\Sigma} r+\mathbf{\Sigma} s+\mathbf{\Sigma} t+\mathbf{\Sigma} r^{\prime}+\mathbf{\Sigma} s^{\prime}+\mathbf{\Sigma} t^{\prime}\right)+\frac{l^{\prime}(l-1)+m^{2}(m-1)+n^{2}(n-1)}{2} \text {. }
\end{aligned}
$$

Il primo sestinomio può anche scriversi nella forma:

$$
\begin{gathered}
l\left(\mathbf{\Sigma}_{\rho}+\mathbf{\Sigma} \rho^{\prime}\right)+m\left(\mathbf{\Sigma} \sigma+\mathbf{\Sigma} \sigma^{\prime}\right)+n\left(\mathbf{\Sigma} \tau+\mathbf{\Sigma} \tau^{\prime}\right) \\
-(l+m+n)\left(\mathbf{\Sigma} \rho^{\prime}+\mathbf{\Sigma}_{\sigma^{\prime}}+\mathbf{\Sigma}_{\tau^{\prime}}\right),
\end{gathered}
$$

ed equivale quindi, per le (6) e $\left(5^{\prime}\right)$, a

$$
\begin{gathered}
l \frac{(m+n)(m+n-1)}{2}+m \frac{(n+l)(n+l-1)}{2}+n \frac{(l+m)(l+m-1)}{2} \\
-(l+m+n) \frac{l(l-1)+m(m-1)+n(n-1)}{2} .
\end{gathered}
$$

Sostituendo quest' espressione, e facendo uso delle $(6),(7)$ e $\left(7^{\prime}\right)$, si trova facilmente che $i l$ grado di $R$ è l $m$ n.

3. Ora supponiamo che le date superficie abbiano in un punto comune 0 le multiplicità $\lambda, \mu, \nu$ risp.: per vedere quante intersezioni cadono in tal punto, collochiamo in 0 il vertice $(0,0,0,1)$ del tetraedro di riferimento assumendo come piano $x_{3}=0$ un piano affatto arbitrario per $0, \mathrm{e}$ cerchiamo con quale esponente si stacchi da $R$ il futtore $x_{3}$.

A tal fine osserviamo che, per l'ipotesi fatta, le forme $A, B, C$ non possono contenere in nessun termine le coordinate $x_{1}, x_{3}, x_{3}$ ad un grado minore di $\lambda, \mu, \nu$ risp., sicchè possiamo porre:

$$
\left.\begin{array}{lll}
a_{i u}=\alpha_{i u} x_{3}{ }^{\lambda-i} x_{4}{ }^{l-\lambda}+\text { potenze superiori di } x_{3}, & (i \leq \lambda) \\
b_{j v}=\beta_{j v} x_{3}{ }^{\mu-j} x_{4-\mu}{ }^{m-\mu} & \text { id. } & (j \leq \mu) \\
c_{k_{v}}=\gamma_{k_{v}} x_{3}{ }^{\gamma-k} x_{4}^{n-\vartheta}+ & \text { id. } & (k \leq \nu),
\end{array}\right\}
$$


dove le costanti $\alpha_{i u}, \beta_{j v}, \gamma_{k_{v}}$ possono anche essere nulle. Ora consideriamo la matrice $H$, e nella sua matrice parziale 1 moltiplichiamo le linee del primo gruppo per $x_{3}^{\mu+\nu-4}$, quelle del secondo per $x_{3}^{\mu+\nu-2}, \ldots$, quelle del $(\mu+\nu-1)^{\text {mo }}$ per $x_{3}$; analogamente nella 2 moltiplichiamo le linee del primo, del secondo,..., del $(x+\lambda-1)^{\mathrm{m} /}$ gruppo ordinatamente per $x_{3}{ }^{{ }+\lambda_{-1}}, x_{3}{ }^{{ }^{+}+\lambda_{-2}}, \ldots, x_{3}$, e nella 3 le linee del primo, del secondo,..., del $(\lambda+\mu-1)^{\mathrm{ms}}$ grippo per $x_{3}^{\lambda_{+} \mu_{-1}}$, $x_{3}{ }^{\lambda+\mu-z}, \ldots, x_{3}$. Eseguendo tali operazioni anche sulle linee di $I I$ che si sono scelte per formare il determinante $\chi$, questo risulta cosi moltiplicato per $x_{3}$ coll' esponente

$$
\sum_{i=1}^{i=\mu+1}(\mu+\nu-i) \rho_{i}+\sum_{i=1}^{i=1}(\nu+\lambda-i) \sigma_{i}+\sum_{i=1}^{i=1}(\lambda+\mu-i) \tau_{i}
$$

Ma, per effetto di quelle operazioni, nella matrice $H$, e quindi anche in $\chi$, ogni colonna del primo, del secondo,..., del $(\lambda+\mu+\nu-1)^{\text {mu }}$ gruppo riesce divisibile risp. per $x_{3}^{\lambda_{+} \mu_{+} \nu_{-1}}, x_{3}{ }^{\lambda_{+} \mu_{+}+-}, \ldots, x_{3}$; e perciò $\chi$ riesce divisibile per $x_{3}$ coll' esponente

$$
\begin{gathered}
(\lambda+\mu+\nu-1)+2(\lambda+\mu+\nu-2)+\cdots+(\lambda+\mu+\nu-2) \cdot 2 \\
+(\lambda+\mu+\nu-1),
\end{gathered}
$$

cioè coll' esponente

$$
\frac{(\lambda+\mu+v)\left[(\lambda+\mu+v)^{2}-1\right]}{6} \text {. }
$$

Risulta quindi posto in evidenza in $\chi$ il fattore $x_{3}$ coll' esponente

$$
\frac{(\lambda+\mu+v)\left[(\lambda+\mu+\nu)^{2}-1\right]}{6}
$$

Per la matrice $H^{\prime}$, moltiplichiamo invece in I' le colonne del primo, del se* condo,..., del $(\nu-1)^{\mathrm{m}}$ gruppo per $x_{3}{ }^{{ }^{\prime}-1}, x_{3}{ }^{{ }^{2}-z}, \ldots, x_{3}$ risp.; in $\mathrm{IL}^{\prime}$ le colonne del primo, del secondo,..., del $(\lambda,-1)^{\mathrm{m}}$ gruppo per $x_{3}{ }^{\lambda-1}, x_{3}{ }^{2}{ }^{2}, \ldots, x_{3} ; \mathrm{e}$ in III' le colonne del primo, secondo, $\ldots,(\mu-1)^{\text {mut }}$ gruppo per $x_{3}{ }^{\mu-1}, x_{3}{ }^{\mu-z}, \ldots, x_{3}$. Eseguendo queste stesse operazioni sopra le colonne di $\chi^{\prime}$, questo risulta moltiplicato per $x_{3}$ coll' esponente

$$
\sum_{i=1}^{i=1} i(\lambda-i)+\sum_{i=1}^{i-1} i(u-i)+\sum_{=1}^{i-1} i(\nu-i),
$$


ciò̀ coll' esponente

$$
\frac{\lambda^{3}+\mu^{3}+\nu^{3}-(\lambda+\mu+\nu)}{6}
$$

$\mathrm{Ma}$, in seguito a quelle operazioni, riescono divisibili, in $1^{\prime}$, le linee del primo, secondo, $\ldots,(\mu+\nu-1)^{\mathrm{m} v}$ gruppo risp. per $x_{3}{ }^{\mu+\nu-1}, x_{3}{ }^{\mu+\nu-2}, \ldots, x_{3} ;$ in $2^{\prime}$, le linee del primo, $\ldots,(\nu+\lambda-1)^{\mathrm{m})}$ gruppo per $x_{3}{ }^{\nu+\lambda-1}, \ldots, x_{3}$; in $3^{\prime}$, le linee del primo $, \ldots,(\lambda+\mu-1)^{\mathrm{m})}$ gruppo per $x_{3}{ }^{{ }^{2}+\mu_{-1}}, \ldots, x_{3}$. Quindi $\chi$ 'riesce divisibile per $x_{3}$ coll' esponente

$$
\sum_{i=1}^{+-1}(\mu+\nu-i) \rho_{i}^{\prime}+\sum_{i=1}^{i=\sum^{+\lambda-1}}(\nu+\lambda-i) \tau_{i}^{\prime}+\sum_{i=1}^{i=i+1-1}(\lambda+\mu-i) z_{i}^{\prime} .
$$

Epperò vien posto in evidenza in $\chi^{\prime}$ il fattore $x_{3}$ coll'esponente

$$
\begin{aligned}
& \sum_{i=1}^{+}(\mu+\nu-i) \rho^{\prime} i+\sum_{i=1}^{i=}(\nu+\lambda-i) \sigma^{\prime}{ }_{i}+\sum_{i=1}^{i=1}(\lambda+\mu-i) r_{i}^{+1} \\
& -\frac{\lambda^{3}+\mu^{3}+\nu^{3}-(\lambda+\mu+\nu)}{6} .
\end{aligned}
$$

Ne segue che la risultante $R$ è divisibile per $x_{3}$ coll' esponente

$$
\begin{aligned}
& \frac{1}{6}\left\{(\lambda+\mu+\nu)^{3}+\lambda^{3}+\mu^{3}+\nu^{3}-2(\lambda+\mu+\nu)\right\}
\end{aligned}
$$

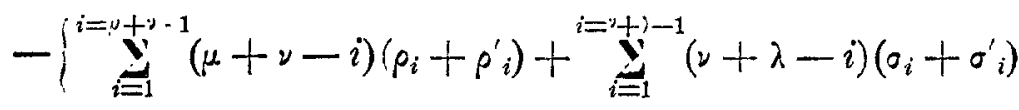

$$
\begin{aligned}
& \left.+\sum_{i=1}^{i=i+1-1}(\lambda+\mu-i)\left(\tau_{i}+\tau_{i}^{\prime}\right)\right\}
\end{aligned}
$$

la qual quantità,. per le (6), si riduce facilmente a $\lambda \mu \nu$. Si conclude che il punto 0 assorbe aramexo $\gamma \mu \nu$ intersezioni delle date superficie.

4. Nel punto 0 sarà racolto un numero d'intersezioni delle tre superficie maggiore di $\lambda . u . \nu$, allora ed allora soltanto che sarà nullo in $R$ il coeffciente di $x_{3}^{{ }^{\prime \mu \nu}}$ : dobbiamo dunque cercare questo coefficiente, e stabilirno il significato geometrico. A tal fine occorre premettere la seguente osservazione. Siano

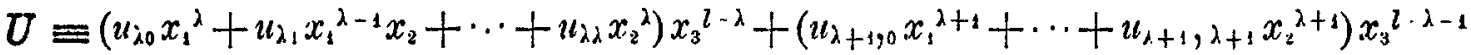

$$
\begin{aligned}
& +\cdots+\left(u_{l} x_{1}^{l}+u_{l} x_{1}^{l-1} x_{2}+\cdots+u_{l l} x_{2}^{l}\right)=0 \text {, } \\
& \nabla \equiv\left(v_{\mu n} x_{1}{ }^{\mu}+\cdots+v_{\mu \mu} x_{2}^{\mu}\right) x_{3}^{m-\mu}+\cdots+\left(v_{m 0} x_{1}^{m}+\cdots+v_{m m} x_{2}^{m}\right)=0 \text {, } \\
& W \equiv\left(u_{y_{0}} x_{1}{ }^{\nu}+\cdots+w_{v} x_{2}{ }^{\nu}\right) x_{3}^{n-\nu}+\cdots+\left(w_{n 0} x_{1}^{n}+\cdots+w_{n n} x_{2}^{n}\right)=0
\end{aligned}
$$


le equazioni di tre curve piane $U, V, W$ degli ordini $l, m, n$, aventi nel punto fondamentale $(0,0,1)$ risp. le moltiplicità $\lambda, \mu, \nu$ : la condizione necessaria e sufficiente perchè esse abbiano in comune un punto diverso dal precedente (ma che potrebbe però anche coincidere con questo, nel senso che esso conti fra $i$ punti comuni alle tre curve più di quello che conta in generale pel solo fatto che esse vi passano $\lambda, \mu, \nu$ volte) si può ottenere come quoziente di due determinanti, analogamente a ciò che si ̀̀ fatto nel n. ${ }^{\circ} 1$, nel modo seguente. Moltiplichiamo le (14) ordinatamente per

$$
\begin{aligned}
& x_{1}^{\mu+\nu-1}, x_{1}^{\mu+\nu-2} x_{2}, \ldots, x_{2}^{\mu+\nu-1} ; \ldots ; x_{1}^{m+n-2}, x_{1}^{m+n-3} x_{2}, \ldots, x_{2}^{m+n-2} ; \\
& x_{1}{ }^{\nu+\lambda-1}, x_{1}{ }^{\nu+\lambda-2} x_{2}, \ldots, x_{2}{ }^{\nu+\lambda-1} ; \ldots ; x_{1}{ }^{n+l-2}, x_{1}{ }^{n+l-3} x_{2}, \ldots, x_{2}{ }^{n+l-2} \text {; } \\
& x_{1}^{\lambda+\mu-1}, x_{1}^{\lambda+\mu-2} x_{2}, \ldots, x_{2}^{\lambda+\mu-1} ; \ldots ; x_{1}^{l+m-2}, x_{1}^{l+m-3} x_{2}, \ldots, x_{2}^{l+m-2} \text {. }
\end{aligned}
$$

Ottenjamo così

$$
\left.\begin{array}{l}
\frac{1}{2}\{(m+n)(m+n-1)+(n+l)(n+l-1)+(l+m)(l+m-1)\} \\
-\frac{1}{2}\{(\mu+\nu)(\mu+\nu-1)+(\nu+\lambda)(\nu+\lambda-1)+(\lambda+\mu)(\lambda+\mu-1)\}
\end{array}\right\}
$$

equazioni lineari fra le potenze

$x_{1}{ }^{2+\mu+\nu-1}, x_{1}{ }^{\lambda+\mu+\nu-2} x_{2}, \ldots, x_{2}{ }^{\lambda+\mu+\nu-1} ; \ldots ; x_{1}^{l+m+n-2}, x_{1}^{l+m+n-3} x_{2}, \ldots, x_{2}^{l+m+n-2}$,

il cui numero è

$$
\frac{1}{2}\{(l+m+n)(l+m+n-1)-(\lambda+\mu+\nu)(\lambda+\mu+\nu-1)\} .
$$

Le equazioni ottenute non sono però fra loro indipendenti, giacchè se moltiplichiamo le identità

$$
V W=W V, \quad W U=U W, \quad U V=V U
$$

risp. per

$$
\begin{aligned}
& x_{1}^{\lambda-1}, x_{1}^{\lambda-2} x_{2}, \ldots, x_{2}^{\lambda-1} ; \ldots ; x_{1}^{l-2}, x_{1}^{l-3} x_{2}, \ldots, x_{2}^{l-2} ; \\
& x_{1}^{\mu-1}, x_{1}^{\mu-2} x_{2}, \ldots, x_{2}^{\mu-1} ; \ldots ; x_{1}^{m-2}, x_{1}^{m-3} x_{2}, \ldots, x_{2}^{m-2} ; \\
& x_{1}{ }^{\nu-1}, x_{1}{ }^{\eta-2} x_{2}, \ldots, x_{2}{ }^{n-1} ; \ldots ; x_{1}^{n-2}, x_{1}^{n-3} x_{2}, \ldots, x_{2}^{n 2} \text {, }
\end{aligned}
$$

otteniamo

$$
\frac{1}{2}\{l(l-1)+m(m-1)+n(n-1)\}-\frac{1}{2}\{\lambda(\lambda-1)+\mu(\mu-1)+\nu(\nu-1)\}
$$


relazioni lineari fra le equazioni stesse, o il numero (17) è la differenza fra i numeri (15) e (16). Se allora si formano due matrici, l'una coi coefficienti delle equazioni, l'altra coi coefficienti delle relazioni da cui esse sono vincolate, la richiesta condizione si ottiene uguagliando a zero il rapporto fra uno qualunque (non jdenticamente nullo) des determinanti tratti dalla prima e il determinante supplementare tratto dalla seconda. Gli ordini dei due determinanti sono dati risp. dalle espressioni (16) e (17).

5. Per riconoscere ormai quale sia in $R$ il coefficiente di $x_{3}{ }^{3 \mu \nu}$, supponiamo di aver eseguito sulle matrici $H, H^{\prime}$, e però an he su $\chi, \chi^{\prime}$, le operazioni indicate al n. ${ }^{\circ} 3$. Il risultato che ricaveremo dall' $R$ primitivo sarà $\frac{R}{x_{3^{j \mu \nu}}}$, sicchè il cercato coefficiente sarà ciò che diventa questo quoziente ponendovi $x_{3}=0$.

In $H$ scompongasi ciascuna delle matrici parziali $1,2,3$ in altre due, che diremo risp. $1_{1}, 1_{2} ; 2_{1}, 2_{2} ; 3_{1}, 3_{2}$, ponendo nelle $1_{1}, 2_{1}, 3_{1}$ risp. $\mathrm{i}$ primi $\mu+\nu, \nu+\lambda, \lambda+\mu$ gruppi di linee di $1,2,3$, e nelle $1_{2}, 2_{2}, 3$, i gruppi rimanenti. Scompongasi jnoltre la $H$ in due matrici I e II, ponendo nella prima i primi $\lambda+\mu+\nu-1$ gruppi di colonne, e nella seconda i gruppi rimanenti. Un elemento qualunque, il quale appartenga ad una colonna del gruppo $j^{\text {mo }}$ di $H$ e ad una linea del gruppo $i^{\text {mo }}$ di 1 , o di 2 , o dı 3 , può rappresentarsi risp. con $a_{j-i, r}, 0$ con $b_{j-i, r}$, o con $c_{j-i, r}$ : vediamo ciò che esso diventa, a seconda delle matrici $1_{1} I, 1_{2} I, \ldots$ a cui appartiene, quando, eseguite le operazioni su accennate, vi si pone $x_{3}=0$.

1 I) Nell'elemento $a_{j-i, r}$ si ha $i \leq \mu+\nu, j \leqslant \lambda+\mu+\nu-1$. Esso è stato moltiplicato per $x_{3}{ }^{\mu_{+}{ }^{-i} i}$ e diviso per $x_{3}{ }^{\lambda_{+} \mu_{+}{ }^{-} j}$, e però in sostanza è stato moltiplicato per $x_{3}^{j-i-\lambda}$. Ora se $j-i \leq \lambda$, in $a_{j-i, r}$ esiste il fattore $x_{3}{ }^{\lambda-j+i}$, sicchè, fatte le precedenti operazioni e posto $x_{3}=0$, si ottiene $a_{j-j-i, r}[\mathrm{v}$. ]e (13)]. Se invece $j-i>\lambda$, si ottiene zero.

$1_{2}$ I) Si ha $i>\mu+\nu, j \leq \lambda+\mu+\nu-1$, da cui segue $j-i<2$, quindi l'elemento $a_{j-i . r}$ contiene il fattore $x_{3}{ }^{\lambda-j+i}$. Esso è stato sultanto diviso per $x_{3}{ }^{\lambda+\mu_{+}+\nu}$, dunque risulta ancora come fattore $x_{3}{ }^{i-\mu_{-\nu}}$, dove l'esponente è $>0$. Per $x_{3}=0$ esso si anrulla.

$2, I)$ L'elemento è $b_{j-i, r}$ : per $j-i \leqslant \mu$ si ottiene $\beta_{j-i, r}$, e per $j-i>\mu$ si ottiene zero.

2, I) Tutti gli elementi si annullano.

3, I) L' elemento è $c_{j-i, r} ;$ per $j-i \leqslant \nu$ si ha $\gamma_{j-i, r}$, per $j-i>v$ risulta zero. 
3. I) Tutti gli elementi si annullano.

Quanto agli elementi della matrice II, essi assumono il valore che acquistano per $x_{3}=0$, all'infuori di quelli che appartengono alle linee dei primi $\mu+\nu-1, \nu+\lambda-1, \lambda+\mu-1$ gruppi di $1,2,3$ risp., $i$ quali si annullano tutti.

Nella $H^{\prime}$ scindasi ciascuna delle matrici $1^{\prime}, 2^{\prime}, 3^{\prime}$ in altre due, che diremo risp. $1_{1}^{\prime}, 1_{2}^{\prime} ; 2^{\prime}, 2_{2}^{\prime} ; 3^{\prime}, 3_{2}^{\prime}$, ponendo nelle $1^{\prime}, 2^{\prime}{ }_{i}, 3^{\prime}$, risp. le linee dei primi $\mu+\nu-1, \nu+\lambda-1, \lambda+\mu-1$ gruppi di $1^{\prime}, 2^{\prime}, 3^{\prime}$, e nelle $1^{\prime}, 2_{2}^{\prime}, 3$ le linee dei gruppi rimanenti. Inoltre dividasi anche ciascuna delle matrici $\mathrm{I}^{\prime}, \mathrm{II}^{\prime}, \mathrm{III}^{\prime}$ in altre due, che risp. diremo $\mathrm{I}_{1}^{\prime}, \mathrm{I}_{2} ; \mathrm{II}_{1}^{\prime}, \mathrm{II}_{2}^{\prime}$; $\mathrm{III}_{1}, \mathrm{III}_{2}^{\prime}$, ponendo nelle $\mathrm{I}_{1}^{\prime}, \mathrm{II}_{1}^{\prime}, \mathrm{III}$, i primi $\nu-1, \lambda-1, \mu-1$ gruppi di colonne di I', II', III', e nelle $\mathrm{I}_{z}^{\prime}$, $\mathrm{II}_{z}^{\prime}$, $\mathrm{III}_{z}^{\prime}$ i gruppi rimanenti. Un elemento qualunque, il quale appartenga all' $i^{m o}$ gruppo di linee di 1 ', o di $2^{\prime}$, o di 3', ed all'jmo gruppo di colonne di I', o di II' a di III', viene rappresentato, prescindendo dal segno, con una delle lettere $a, b, c$, affetta da due indici, di cui il primo è $i-j$ : eseguite le operazioni di cui sopra si è parlato, e posto $x_{3}=0$, con ragionamenti del tutto analoghi ai precedenti si giunge al seguente risultato:

$$
\begin{aligned}
& \text { gli elementi di }\left(1^{\prime}, I_{i}^{\prime}\right) \text { diventano }-\beta_{i-j, r} \text { se } i-j \leqslant \mu \text {, } \\
& \text { " } \quad "\left(2^{\prime}{ }_{1} \mathrm{I}_{1}\right) \quad " \quad \alpha_{i-j, r} \quad n \quad i-j \leq \lambda \text {, } \\
& n \quad " \quad\left(2^{\prime}, \mathrm{II}^{\prime}{ }_{1}\right) \quad n \quad-\gamma_{i-j, r} \quad n \quad i-j \leq \nu \text {, } \\
& \text { " } "\left(3_{1}^{\prime} I_{1}^{\prime}\right) \quad n \quad \beta_{i-j, r} \quad n \quad i-j \leq \mu \text {, }
\end{aligned}
$$

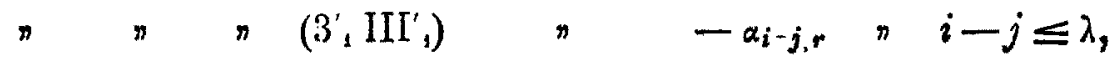

$$
\begin{aligned}
& n \quad " \quad\left(1_{1}^{\prime} \mathrm{III}_{1}^{\prime}\right) \quad \text { " } \quad \text { i-j,r, } n \quad i-j \leqslant \nu \text {, }
\end{aligned}
$$

mentre si annullano in caso contrario;

tutti gli elementi di $\left(\mathrm{I}_{1}^{\prime} \mathrm{I}_{2}^{\prime}\right)$ e $\left(2^{\prime}{ }_{1} \mathrm{I}_{2}\right)$, di $\left(2^{\prime}{ }_{1} \mathrm{II}_{2}^{\prime}\right)$ e $\left(3^{\prime}{ }_{1} \mathrm{II}_{2}^{\prime}\right)$, di $\left(3^{\prime}{ }_{1} \mathrm{III}_{2}\right)$ c $\left(I^{\prime}, I I I_{2}\right)$ si annullano, salvo se si ha ordinatamente $j=\nu, j=\lambda, j=\mu_{\text {, }}$ nei quali casi essi diventano $-\beta_{i-\gamma, r}$ e $\alpha_{i-\gamma, r},-\gamma_{-\lambda, \nu}$ e $\beta_{i-\lambda, r},-\alpha_{i-\mu, r}$ e $\% i \cdot \mu, r ;$

tutti gli elementi di $\left(1_{2}^{\prime} \mathrm{I}_{2}^{\prime}\right),\left(2_{2}^{\prime} \mathrm{I}_{2}^{\prime}\right),\left(2_{2}^{\prime} \mathrm{II}_{2}^{\prime}\right),\left(3_{2}^{\prime} \mathrm{II}_{2}^{\prime}\right),\left(3_{2}^{\prime} \mathrm{III}_{2}^{\prime}\right),\left(1_{2}^{\prime} \mathrm{III}_{2}^{\prime}\right)$ acquistano il ralore che essi assumono per $x_{3}=0$;

infine tutti gli elementi delle dieciotto matrici rimanenti si annullano. 6. Per brevità, chiamiamo $D$ e $\Delta$ i due termini della differenza (15), $T$ e $\Theta$ quelli della (16). Cio posto, consideriamo di nuoro la $H$ : abbiamo 
veduto che nella sua matrice $I I$, in seguito alle fatte operazioni, si sono annullati tutti gli elementi di $\Delta$ linee; ma il numero totale delle linee di $H$ è $D$, quindi $D-\Delta$ esprime il numero delle linee di II, ciascuna delle quali è risultata composta, in generale, di elementi non tutti nulli. Il numero delle colonne di II è invece $T-\Theta$. Siccome è

$$
D-\Delta \geqslant T-\Theta \text {, }
$$

per formare il determinante $\chi$ potremo scegliere $T$ linee di $H$, delle quali $T-\Theta$ non siano risultate composte, nella loro parte contenuta in II, di elementi tutti nulli, e le rimanenti $\Theta$ siano fra quelle (il cui numero totale è $\Delta$ ) che in II sono diventate di tutti elementi nulli. Se allora si pensa $\chi$ scomposto in due matrici, di cui l'una formata colle colonne dei primi $\lambda+\mu+\nu-1$ gruppi (cioè colle prime $\Theta$ colonne), e l'altra furmata colle $T-\Theta$ colonne rimanenti (cioè con quelle che appartengono a II), è chiaro che, eseguite le solite operazioni, colle linee della seconda matrice non si può formare che un solo determinante non nullo. Laonde $\chi$ per tali operazioni diventa il prodotto di due determinanti $\chi_{1}$ e $\chi_{2}$ : il primo, di ordine $\Theta$, ha le sue linee tratte dai primi $\mu+\nu-1, \nu+\lambda-1, \lambda+\mu-1$ gruppi di 1 I, 2 I, 3 I, ed ha per elementi le $x, \beta, \gamma$ risp.; il secondo, di ordine $T-\Theta$, ha le sue linee tratte dagli $(m+n-1)-(\mu+\nu-1)=m+n-\mu-\nu, n+l-\nu-\lambda$, $l+m-\lambda-\mu$ gruppi che in II non sono risultati di tutti elementi nulli, ed ha per elementi $\mathrm{i}$ valori che si ottenqono ponendo $x_{3}=0$ nei corrispondenti elementi di II, i cui primi indici non sono minori di $\lambda, \mu . \nu$ risp.

Con ciò che precede, la composizione del determinante $\chi^{\prime}$ è già del tutto fissata; ed eseguite le solite operazioni, $\Delta-\Theta$ fra le sue lince sono formate cogli elementi $\alpha, \beta, \%$, e le altre, in numero di $(D-\Delta)-(T-\theta)$, sono formate coi risultati che s'ottengono ponendo $x_{3}=0$ negli elementi $a, b, c$ i cui primi indici non siano risp. minori $d i \lambda, \mu, \nu$. Se quindi pensiamo $\chi^{\prime}$ scomposto in due matrici, di cui l'una formata colle linee della prima specie e l'altra colle rimanenti, eseguite le solite operazioni, dnlla seconda non si pud trarre che un solo detcrminante non nullo; sicchè per tali operazioni $\chi^{\prime}$ si scinde nel prodotto di due determinanti $\chi^{\prime}$, e $\chi_{2}^{\prime}$, di ordini $\Delta-\Theta$ e $(D-\Delta)-(T-\Theta)$.

Il coefficiente di $x_{3}^{{ }^{\lambda \mu \nu}}$ in $R$ è dunque il prodotto $\frac{\chi_{4}}{\bar{\chi}_{4}^{\prime}} \cdot \frac{\chi_{2}}{\chi_{2}^{\prime}}$, e la ricerca del significato geometrico dell' annullarsi de' suoi due fattori non offre ormai nessuna difficoltà. Invero si osservi che $\mathrm{i}$ coni $\mathrm{d}$ 'ordini $\lambda, \mu, \nu$ tangenti alle 
tre superficie $A, B, C$ nel punto singolare 0 hanno per equazioni:

$$
\begin{aligned}
& \alpha_{00} x_{3}{ }^{\lambda}+\left(\alpha_{10} x_{1}+\alpha_{11} x_{2}\right) x_{3}{ }^{\lambda-1}+\cdots+\left(\alpha_{\lambda .0} x_{1}{ }^{\lambda}+\cdots+\alpha_{2 \lambda} x_{2}{ }^{\lambda}\right)=0 \text {, } \\
& \beta_{00} x_{3}{ }^{\mu}+\text {. . . . . . . . . . . . . . . . . . . . }=0 \text {, } \\
& \gamma_{00} x_{3}{ }^{\nu}+
\end{aligned}
$$

mentre le sezioni delle superficie stesse col piano $x_{3}=0$ sono rappresentate da:

$\left(a_{\lambda 0}^{\prime} x_{1}^{\lambda}+a_{\lambda_{1}}^{\prime} x_{1}^{\lambda-1} x_{2}+\cdots+a_{\lambda \lambda}^{\prime} x_{2}^{\lambda}\right) x_{l}^{l-\lambda}+\cdots+\left(a^{\prime}{ }_{l 0} x_{1}^{l}+\cdots+a_{l l}^{\prime} x_{2}^{l}\right)=0$, $\left(b_{\mu, 0}^{\prime} x_{1}{ }^{\mu}+\right.$. . . . . $\left.+U_{\mu \mu}^{\prime} x_{2}{ }^{\mu}\right) x_{1}{ }^{m-\mu}+$. . . . . . . . . $=0$,

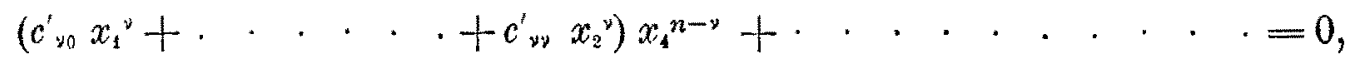

dove le $a^{\prime}, b^{\prime}, c^{\prime}$ denotano i coefficienti dei termini indipendenti da $x_{3}$ nelle corrispondenti forme $a, b, c$ (onde le $a_{\lambda i}^{\prime}, b_{\mu i}^{\prime}, c_{y_{i}}^{\prime}$ coincidono risp. colle $\alpha_{\lambda i}$, $\left.\beta_{\mu_{i}}, \gamma_{v i}\right)$. Allora il primo fattore $\frac{\chi_{1}}{\chi_{1}^{\prime}}$ ̀े costituito, colle $\alpha, \beta, \gamma$ delle (18), nell'identico modo col quale si disse nel n. 1 doversi formare, colle $a, b, c$ delle (1), il quoziente $\frac{\chi}{\chi^{\prime}}$ : uguagliato a zero, esso rappresenta dunque la risultante dell'eliminazione dei rapporti $\frac{x_{1}}{x_{3}}, \frac{x_{2}}{x_{3}}$ dalle (18), ciò̀ la condizione necessaria e sufficiente perchè $i$ coni tangenti in 0 alle tre date superficie $a b-$ biano una generatrice comune. L'annullarsi del secondo fattore $\frac{\chi_{2}}{\chi^{\prime}}$ è invece (n. ${ }^{\circ}$ 4) la condizione perchè le sezioni fatte nelle date superficie dal piano $x_{3}=0$ abbiano in comune un punto distinto da 0 . Per l'ipotesi fatta che le tre superficie non passino per una medesima curva, e per il modo del tutto arbitrario con cui abbiamo scelto la faccia $x_{3}=0$ del tetraedro fondamentale, quest'ultimo fatto certamente non ha luogo, sicchè il secondo fattore è essenzialmente diverso da zero. Ciò dimostra la proposizione enunciata in principio.

7. Possiamo spingere la ricerca più oltre. I coni tangenti in 0 alle tre superficie abbiano una generatrice comune $g$, e per maggior generalità poniamo che questa abbia raccolte in 0 risp. $\lambda+\xi, \mu+\eta, \nu+\zeta$ delle sue intersezioni colle superficie $A, B, C$ : sarà

$$
\xi \geqslant 1, \quad n \geqslant 1, \quad \xi \geqslant 1,
$$


e possiamo supporre che sia, per es.,

$$
\xi \leq n \leq \zeta \text {. }
$$

Assumiamo $g$ come retta $x_{1}=x_{2}=0$, di guisa che nelle (18) si arrà

$$
\alpha_{00}=\beta_{00}=\gamma_{00}=0 \text {. }
$$

Inoltre, se nelle (1) si pone $x_{1}=x_{3}=0$, si devono risp. staccare dai primi membri i fattori $x_{3}{ }^{\lambda+\xi}, x_{3}{ }^{\mu+\eta}, x_{3}{ }^{\nu+\zeta}$, onde potremo scrivere:

e sarà

$$
\begin{array}{ll}
a_{00}=p x_{3}{ }^{\lambda+\xi} x_{1}^{l-\lambda-\xi}+\text { potenze superiori } \mathrm{di} x_{3}, \\
b_{00}=q x_{3}{ }^{\mu+\eta} x_{1}^{m-u-\eta}+\cdots \\
c_{00}=r x_{3}^{\nu+\xi} x_{4}^{n-\nu-\xi}+\cdots x_{3},
\end{array}
$$

$$
p=0, \quad q=0, \quad r=0 \text {. }
$$

Volendo cercare quante intersezioni delle $A, B, C$ sono assorbite dal punto 0 , per semplicità, poniamo nel determinante $\chi$ la prima linea di ciascuna delle matrici $1,2,3$ di $H$ : ciò che, per i numeri precedenti, è sempre lecito. Allora, rifacendo il ragionamento del $n .^{\circ} 3$, si trova che la prima colonna di $H$, e però anche di $\chi$, riesce divisibile non soltanto per $x_{3}^{\lambda+\mu+\nu-1}$, ma per $x_{3}^{\lambda+\mu+\nu-1+\xi}$, sicchè da $\chi$ si stacca il fattore $x_{3}$ con un esponente superiore di almeno $\xi$ unità a quello che averasi nel $n .^{\circ} 3$, mentre l'esponente della potenza di $x_{3}$, che entra come fattore di $\chi^{\prime}$, non è ora diverso da quello di prima. Dunque il punto 0 assorbe asmeno $\lambda \mu \nu+\xi$ intersezioni delle date superficie.

In 0 cadrà un maggior numero di tali intersezioni quando sarà nullo in $R$ il coefficiente di $x_{3}^{\lambda \mu \nu+\xi}$. Per trovare questo coefficiente, rifacciamo esattamente tutte le operazioni descritte nel n. 3 : soltanto, poichè abbiamo già notato che la prima colonna di $\chi$ riesce ora divisibile, anzichè per $x_{3}^{\lambda+\mu+8-1}$, per $x_{3}^{\lambda+\mu+\nu-1+\xi}$, converrà dividerla appunto per questa potenza. Poscia porremo $x_{3}=0$. Il risultato sarà allora, come nel numero precedente, il prodotto di due quozienti di determinanti, dei quali il secondo è lo stesso $\frac{\chi_{2}}{\chi^{\prime}}$, e, per le ragioni già addotte, è essenzialmente diverso da zero. Il primo quoziente invece si deduce da $\frac{\chi_{1}}{\chi_{1}^{\prime}}$ eseguendo su esso alcune modificazioni, che variano a seconda dei casi; e precisamente: secondo che è $\xi<n$ (e del resto $n \leq \zeta$ ), 
oppure $\xi=n<\zeta$, oppure $\xi=\eta=\zeta$, nella prima colonna del numeratore, in luogo di $\alpha_{00}, \beta_{00}, \gamma_{00}$ vengono risp. i valori $p, 0,0$, oppure $p, q, 0$, oppure $p, q, r$, mentre in ogni caso in tutte le colonne rimanenti del numeratore, come pure dappertutto nel denominatore, in luogo di $\alpha_{00}, \beta_{00}, \gamma_{00}$ compare lo zero.

Scomponendo quindi il numeratore in due matrici, costituite l'una delle prime tre colonne e l'altra delle rimanenti, colle linee della prima non si può formare che un determinante non nullo, ciò̀, risp. nei tre casi dianzi accennati,

$$
\left|\begin{array}{lll}
p & \alpha_{10} & \alpha_{11} \\
0 & \beta_{10} & \beta_{11} \\
0 & \gamma_{10} & \gamma_{11}
\end{array}, \quad\right| \begin{array}{lll}
p & \alpha_{10} & \alpha_{11} \\
q & \beta_{10} & \beta_{11} \\
0 & \gamma_{10} & \gamma_{11}
\end{array}|, \quad| \begin{array}{lll}
p & \alpha_{10} & \alpha_{11} \\
q & \beta_{10} & \beta_{11} \\
r & \gamma_{10} & \gamma_{11}
\end{array} \mid .
$$

Il detarminante sunnlementare teatto dall' altra matrice è ciǹ she rimane 
Ora diciamo $A^{\prime}$ la superficie che ha per equazione

$$
\Lambda^{\prime} \equiv p z^{\xi_{+1}}+\alpha_{10} x+\alpha_{11} y=0,
$$

e ehe è percio una superficie particolare del fascio rappresentato, al variaro di $k$, da

$$
h: z^{\xi+1}+\alpha_{10} x+\alpha_{11} y=0 .
$$

Se chiamiamo $\Gamma_{A}$ il cono tangente ad A noll'origine, e $\pi$ il piano tangente a $\Gamma_{A}$ lungo l'asse delle $z$, ciò̀ quello che ha per equazione

$$
\pi \equiv \alpha_{10} x+\alpha_{11} y=0,
$$

uma superficie qualunque del fascio (22) è un cilindro di grado ${ }_{-}+1$, le cui generatrici sono parallele all'intersezione del piano $\pi$ col piano $x y$, e di cui la retta all' infinito del piano $x y$ è una generatrice multipla secondo $s$, colla particolarita che $\mathrm{i} \xi$ piani tangenti lungo essa al cilindro coincidono tutti col piano all'infinito dello spazio. Oltre a ciò, tutti i cilindri del fascio sono secati dal piano $\pi$ in $\xi+1$ generatrici coincidenti colla traccia di quel piano sul piano $x y$. Al fascio appartengono, come cilindri degeneri, il piano $x y$ contato $\xi+1$ volte, ed il piano $\pi$ insieme col piano all'infinito contato volte, sicchè, fissato il piano coordinato $x y$ (nonchè il piano $x_{1}=0$ ), il fascio può ritenersi perfettamente noto: la sua base si compone della retta all'infinito del piano $x y$ contata $\zeta(\xi+1)$ volte, e dell'intersezione dei piani $x y$ e $\pi$, contata $\xi+1$ volte.

Per definire, entro il fascio, il cilindro particolare $A^{\prime}$, si consideri un piano qualunque parallelo all' asse delle $z$ (cioè alla $g$ ) e infinitamente vicino all'origine (cioè alla $g$ ): piano la cui equazione sarà quindi della forma

$$
\varepsilon_{1} x+\varepsilon_{2} y=\varepsilon,
$$

dove $\varepsilon_{1}, \varepsilon_{3}$ sono costanti arbitrarie, ed $\varepsilon \dot{e}$ un infinitesimo. Volendo determinare $\mathrm{i}$ punti che, essendo comuni a questo piano ed alle superficie $A$ e $A^{\prime}$, cadono in prossimità dell' origine, basta ricavare dalle (22) e (23) le $x$, y e sostituirle nell'equazione (20) di $A$. Si giunge cosi alla seguente equazione in $z$ :

$$
(p-k) z^{\lambda+\xi}+\cdots=0
$$

dove $\mathrm{i}$ termini che non si sono scritti o contengono potenze di $z$ superiori alla $(\lambda+\xi)^{m a}$, o dipendono da $\varepsilon$. Se dunque si vuole che per $\varepsilon$ infinitesimo cada in 0 il massimo numero di quelle intersezioni, bisogna porre $k=p$, il 
che fornisce, nel fascio (22), appunto la superficie $A^{\prime}$. Per la proprietà ehe ora le abbiamo riconosciuta, e che la caratterizza fra gli elementi del fascio, chiameremo $A^{\prime}$ la superficie cilindrica osculatrice all $A$ e corrispondente alla generatrice $g$ del cono $\Gamma_{A}\left({ }^{*}\right)$.

9. Siamo ora in grado di assegnare il significato geometrico dell' annullarsi dei determinanti (19).

$10^{\circ}$ caso. Quando sia $弓<n$ (ed $\left.n \leq \zeta\right)$, si deve considerare il primo di quei determinanti, il quale equivale a

$$
p\left(\beta_{10} \gamma_{11}-\beta_{11} \gamma_{10}\right) \text {, }
$$

e poichè (n. ${ }^{\circ}$ 7) $p$ non è nullo, resta soitanto da interpretare la condizione

$$
\beta_{10} \gamma_{11}-\beta_{11} \gamma_{10}=0 \text {. }
$$

Ora, se non si ha $\beta_{10}=\beta_{14}=0$, nè $\gamma_{10}=\gamma_{11}=0$, la generatrice $g$ è semplice per ciaseuno dei coni $\Gamma_{B}$ e $\Gamma_{C}$ tangenti in 0 alle superficje $B$ e $C$ risp., e la condizione precedente esprime che i piani tangenti a tali coni lungo $g$ (piani le cui equazioni sono

coincidono.

$$
\left.\beta_{10} x_{1}+\beta_{1:} x_{2}=0, \quad \gamma_{10} x_{1}+\gamma_{11} x_{2}=0\right)
$$

2. caso. Se $\xi=\eta<\zeta$, il secondo dei determinanti (19) si annulla sia quando si ha $\gamma_{s 0}=y_{11}=0$, sia quando è ad un tempo

$$
q \alpha_{20}-p \beta_{10}=0, \quad q \alpha_{11}-p \beta_{13}=0
$$

Nel primo caso il cono $\Gamma_{C}$ ha in $g$ una generatrice almeno doppia; nel secondo caso le superficie eilindriche $A^{\prime}$ e $B^{\prime}$ osculatrici alle $A$ e $B$ e corrispondenti alla $g$ coincidono. Esclusi questi oasi, i due piani

$$
\gamma_{10} x+\gamma_{11} y=0, \quad\left(q \alpha_{10}-p \beta_{10}\right) x+\left(q \alpha_{11}-p \beta_{11}\right) y=0
$$

riescono ben determinati, e l'annullarsi del determinante considerato è la condizione perchè essi coincidano. Il primo è quello che tocea $\Gamma_{C}$ lungo $g$. Per riconoscere che piano sia il secondo, si osservi che le $A^{t}$ e $B^{\prime}$ hanno per equazioni

$$
A^{\prime} \equiv p z^{\xi+1}+\alpha_{10} x+\alpha_{11} y=0, \quad B^{\prime} \equiv q z^{\xi+1}+\beta_{10} x+\beta_{11} y=0 .
$$

(') Non sí dimenticht poro cle la $A^{\prime}$ non à unica, sibbene è tale ogniciualvolta siasi flssato il sistoma lei piani coordinati (cosi one 0 sia l'origine e la generntrice $g$ l'asso dolle $z$. In tatto il seguito si sotintenderi sempre che cio sia stato fatto. 
Ma si ha identicamente

$$
q A^{\prime}-p B^{\prime}=\left(q \alpha_{10}-p \beta_{10}\right) x+\left(q \alpha_{14}-p \beta_{11}\right) y
$$

epperò l'intersezione di $A^{\prime}$ e $B^{\prime}$ si compone della retta all'infinito del piano $x y$ contata $\xi(\xi+1)$ volte e di una curva piana [razionale, ecc. $\left.*^{*}\right)$ di ordine $\xi+1$, situata nel piano rappresentato appunto dalla seconda delle (24). La condizione cercata è dunque che il piano di questa curva coincida col piano tangente al cono $\Gamma_{6}$ lungo la retta $y$.

3. caso. Se $\xi=n=\zeta$, l'annullarsi del terzo fra $i$ determinanti (19) dà la condizione perchè i cilindri $A^{\prime}, B^{\prime}, O^{\prime}$ appartengano ad un medesimo fascio [la cui curva base è composta della retta all'infinito del piano $x y$ contata $\xi(\xi+1)$ volte e di una curva piana razionale d'ordine $\xi+1$, come si \& detto nel caso precedente].

Riassumendo, possiamo enunciare il teorema:

Se tre superficie qualunque $A, B, C$ di ordini $l, m, n$ (non passanti

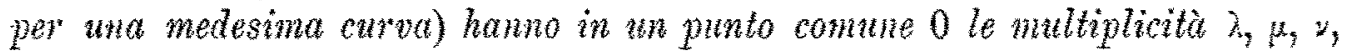
e se $i$ tre coni $\Gamma_{A}, \Gamma_{D}, \Gamma_{C}$ ad esse tangenti in 0 hanno in comme una generatrice $g$, la quale abbia raccolte in 0 rispettivamente $\lambda+\xi, \mu+n_{2} \nu+\zeta$ delle sue intersezioni colle date superficie (essendo, ad esempio, $\xi \leqslant n \leqslant \zeta$ ), qualunque siano le ulteriori singolarita di queste ultime, esse si tagliano in Imn punti, di cui almeno $\lambda \mu \nu+\xi$ cadono in 0. - Q Questo punto assorbe un maggior numero di tali intersezioni soltanto quando $i$ tre coni $\Gamma_{A}, \Gamma_{B}, \Gamma_{C}$ abbiano in comme una seconda generatrice (che può anche coincidere con la $g$, la quale assorbe in tal caso due generatrici di ciascuno di quei coni); oppure:

1) essendo $\leftrightarrows{ }_{n}(e d n \leqslant \zeta)$, quando $g$ sia almeno doppia per $\Gamma_{B} \circ$ per $\Gamma_{C}$, o quando, essendo essa semplice per entrambi $i$ coni, questi abbiano lungo g lo stesso piano tangente;

(*) Si trova tuciluente die, se si jous

$$
M=\frac{q \alpha_{11}-p \beta_{11}}{z_{10} \beta_{11}-\alpha_{11} \beta_{10}}, \quad Y=-\frac{q x_{10} \cdots p \beta_{10}}{x_{10} \beta_{11}-\alpha_{11} \beta_{10}},
$$

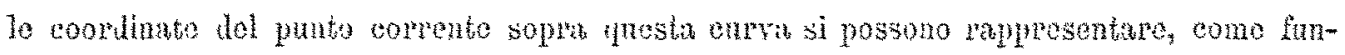
zioni dit un futpanetro $t$, collo formolo:

$$
x=M t^{2}+1, \quad y=N b^{2}+1, \quad z=b
$$

Se poi si assmo como pikno $x=$ quollo della cneva, l'onuaziono di fuesto nel proprio firano 0 :

$$
\left(\alpha_{10} \beta_{11}-\alpha_{11} \beta_{10}\right) x^{*}-\left(z_{12}-1 \beta^{x_{11}}\right) z^{*}+1=0
$$


2) essendo $\%=n<\zeta$, quando, mettendo in evidenza $i$ termini di grado più basso nelle equazioni delle tre superficie

si abbia

$$
\begin{aligned}
& p z^{\lambda+\xi}+\cdots+\left(\alpha_{10} x+\alpha_{11} y\right) z^{\lambda-1}+\cdots=0 \\
& q z^{\mu+\eta}+\cdots+\left(\beta_{10} x+\beta_{11} y\right) z^{\mu-1}+\cdots=0 \\
& r z^{y+\zeta}+\cdots+\left(\gamma_{10} x+\gamma_{11} y\right) z^{y-1}+\cdots=0
\end{aligned}
$$

$$
\mid \begin{array}{lll}
p & \alpha_{10} & \alpha_{11} \\
q & \beta_{10} & \beta_{11} \\
0 & \gamma_{10} & \gamma_{11}
\end{array}=0
$$

cioè quando il cono $\Gamma_{c}$ abbia in g una generatrice almeno doppia, o quando le superficie cilindriche osculatrici alle $A$ e $B$ e corrispondenti alla generatrice $g$ (coincidano, ovvero) si taglino (oltre che nella generatrice $\xi-p l a$ comune) in tha linea (necessariamente piana) situata nel piano tangente a $\Gamma_{U}$ lungo $g$;

3) essendo $\xi=\eta=\zeta$, quando si abbia

$$
\left|\begin{array}{lll}
p & \alpha_{10} & \alpha_{11} \\
q & \beta_{10} & \beta_{11} \\
r & \gamma_{10} & \gamma_{11}
\end{array}\right|=0
$$

ciò̀ quando le superficie cilindriche osculatrici alle $A, B, C$ e corrispondenti alla generatrice $g$ appartengano ad un medesimo fascio.

Per dare un esempio, una delle tre superficie abbia in 0 un punto $h$-planare cogli $h$ piani tangenti coincidenti in un solo $\sigma$; le altre due superficie abbiano in 0 le multiplicità $k$ e $t$, ed i rispettivi coni tangenti $\Gamma$ e $\Gamma$, abbiano in comune una (sola) generatrice $g$ situata in $\sigma$ ed avente risp. $h+h^{\prime}, k+k^{\prime}, t+t^{\prime}$ delle sue intersezioni colle tre superficie raccolte in 0 . Supposto, per fissar le idee, $k^{\prime} \leqslant t^{\prime}$, non potranno accadere che due casi:

1) Se $h^{\prime} \leqq k^{\prime}\left(\leq t^{\prime}\right)$, in 0 cadranno almeno $h k t+h^{\prime}$ intersezioni delle tre superficie, e ne cadrà precisamente questo numero, salvo quando $g$ sia almeno doppia per l'uno o per l'altro dei coni $\Gamma$ e $\Gamma_{1}$, oppure quando, essendo $g$ semplice per entrambi, questi si tocchino lungo $g$;

2) se invece $h^{\prime}>t^{\prime}\left(\equiv k^{\prime}\right)$, oppure se $t^{\prime} \geqslant h^{\prime}>k^{\prime}$, cadranno in 0 almeno $h k t+k+1$ di quelle intersezioni. 
10. Dei risultati a cui siamo giunti si potrebbero fare molteplici applicazioni: qui mi limiterò a qualcuna relativa alla classe di una superficie algebrica (priva di linee multiple). Se questa, che dirò $F$, è di ordine $n$ e priva di punti multipli, la sua classe è $n(n-1)^{2}$, cioè per una retta qualunque si possono condurre ad $F n(n-1)^{2}$ piani tangenti: i loro punti di contatto sono quelli che $F$ ha in comune colle prime polari $F_{1}$ ed $F_{2}$ di due punti arbitrari $P_{1}$ e $P_{2}$ di quella retta. Se invece $F$ è dotata di un punto $s$-plo $(s>1) 0$, quelle prime polari hanno in 0 la multiplicità $s-1$, onde la classe vien diminuita di $s(s-1)^{2}$ unità almeno: proposizione notissima. Ora possiamo vedere in qual caso la diminuzione dovuta al punto $s$-plo sarà maggiore. In virtù del teorema dimostrato nei $n .^{i} 5$ e 6 , perchè ciò avvenga occorre e basta che $\mathrm{i}$ coni $\Gamma, \Gamma_{1}, \Gamma_{2}$ tangenti in 0 alle $F, F_{1}, F_{2}$ abbiano una generatrice comune $g$. Ma $\Gamma_{1}$ e $\Gamma_{2}$ sono le prime polari delle rette $O P_{1}$ ed $O P_{z}$ rispetto al cono $\mathrm{T}$, sicchè la polare di prim'ordine di $g$ rispetto a $\mathrm{I}$ ¿̀ allora indeterminata, epperò $g$ è multipla per $\Gamma$. Risulta dunque dimostrato rigorosamente e per via puramente algebrica il teorema $\left(^{*}\right)$ :

Affinchè un punto s-plo di una superficie d'ordine $n$ (priva di linee multiple) dimimuisca la classe $n(n-1)^{2}$ della superficie di piu che $s(s-1)^{2}$ unità, è necessario e sufficiente che il cono tangente alla superficie nel punto s-plo abbia una generatice multiplo.

Consideriamo più da vicino il caso, in cui il cono $\Gamma$ ha una generatrice $g$ doppia, e per maggior generalità poniamo che questa abbia $s+1+t(t \geqslant 0)$ delle sue intersezioni con $F$ raccolte nel punto 0 . Assumendo 0 come origine delle coordinate o $g$ come asse delle $z$, l'equazione di $F$ può scriversi nella forma :

$$
\begin{aligned}
& F \equiv p z^{s+t+1}+\text { potenze superiori di } z \\
& +x\left(a z^{s+h}+\text { potenze superiori di } z\right)+y\left(b z^{s+z}+\text { potenze superiori di } z\right) \\
& +\left(\alpha_{20} x^{2}+\alpha_{2} x y+\alpha_{2:} y^{3}\right) z^{s-2} \\
& + \text { termini che in } x \text { e } y \text { sono di } 2 .^{\circ} \text { grado e in } z \text { di grado superiore } \\
& \quad \text { all' }(s-2)^{\text {ma }} \\
& \quad+\text { termini di grado superiore al } 2 .^{\circ} \text { in } x \text { e } y=0 .
\end{aligned}
$$

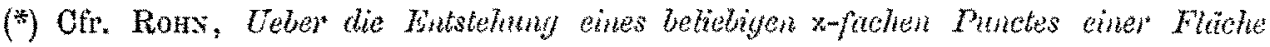

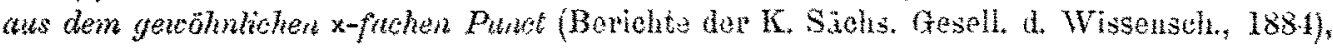
pag. 2 . 
Qui è $p=0, h \geqslant 0, k \geqslant 0$, ma $\mathrm{i}$ termini lineari in $x$ ed $y$ possono anche mancare (onde $a$ e $b$ possono anche essere nulli); invece, poichè l'equazione

$$
\alpha_{20} x^{2}+\alpha_{21} x y+\alpha_{22} y^{2}=0
$$

rappresenta $i$ due piani tangenti a $\Gamma$ lungo $g$, possiamo supporre che nessuna delle $\alpha_{30}, \alpha_{21}, \alpha_{22}$ sia nulla.

Per riconoscere la diminuzione prodotta dal punto 0 nella classe di $F$, consideriamo le intersezioni di $F$ colle prime polari di due punti generici $P$ e $Q$ aventi le coordinate $P_{1}, P_{2}, P_{3}$ e $Q_{1}, Q_{2}, Q_{3}$. Scrivendo soltanto i termini di grado più basso, tali polari hanno per equazioni

$$
\begin{gathered}
a P_{1} z^{s+h}+b P_{2} z^{s+k}+p P_{3}(s+t+1) z^{s+t}+\cdots \\
+\left\{\left(2 \alpha_{30} P_{1}+\alpha_{21} P_{2}\right) x+\left(\alpha_{21} P_{1}+2 \alpha_{22} P_{2}\right) y\right\} z^{s-z}+\cdots=0
\end{gathered}
$$

e quella che di qui si deduce cambiando le $P$ nelle $Q$. Ora dobbiamo distinguere due casi.

1) Se $t$ non ̀̀ maggiore di nessuno dei numeri $h$ e $k$, oppure se mancano nella (25) i termini lineari in $x$ e $y$ (onde $a=b=0$ ), ciascuna delle polari considerate ha raccolte in 0 (in generale ed almeno) $s+t$ delle sue intersezioni con $g$, eppero, colle notazioni del n. prec, abbiamo:

$$
\lambda=s, \quad \mu=\nu=s-1 ; \quad \xi=t+1, \quad \eta \geqslant t+1, \quad \zeta \geqslant t+1 .
$$

Il punto 0 assorbe quindi $s(s-1)^{2}+t+1$ intersezioni delle tre superficie, e non ne assorbe un numero maggiore se non quando sia

$$
0=p\left|\begin{array}{ll}
2 \alpha_{20} P_{1}+\alpha_{21} P_{2} & \alpha_{21} P_{1}+2 \alpha_{22} P_{2} \\
2 \alpha_{20} Q_{1}+\alpha_{21} Q_{2} & \alpha_{21} Q_{1}+2 \alpha_{22} Q_{2}
\end{array}\right|=p\left|\begin{array}{c}
P_{1} P_{2} \\
Q_{1} Q_{2}
\end{array}\right| \cdot\left|\begin{array}{rr}
2 \alpha_{23} & \alpha_{21} \\
\alpha_{21} & 2 \alpha_{22}
\end{array}\right|
$$

Il primo fattore $p$ non è nullo; il secondo fattore si annulla soltanto allorchè la retta $P Q$ taglia l'asse delle $z$, il che è da escludersi avendo scelto $P$ e $Q$ in posizione generica; infine l'annullarsi dell'ultimo fattore significa che $g$ è per $\Gamma$ una generatrice cuspidale.

2) Se $t$ supera quello dei due numeri $h$ e $k$ che non è maggiore dell'altro, e che supporremo, ad es., sia $h$ (cioè se, supposto $h \leq k$, è $h<t$ ), oppure se, mancando uno dei numeri $h$ e $k$ (per es. $k$ ), l'altro è minore di $t$, il più piccolo dei numeri che nel numero precedente abbiamo indicato con $\xi, n, \zeta$ è $h+1$, epperò, tenendo presente che il cono $\Gamma$ ha in $g$ una genera- 
trice doppia, si conclude che 0 assorbe almeno

$$
s(s-1)^{2}+(h+1)+1, \text { ciò̀ } s(s-1)^{2}+h+2
$$

intersezioni delle tre superficie.

Ora nel caso 1) il numero $s+t$ esprime quante fra le intersezioni della retta $g$ colla prima polare di un punto generico dello spazio rispetto ad $F$ cadono in 0 ; nel caso 2 ) invece, 0 rappresenta $s+h$ di tali intersezioni. Abbiamo dunque il teorema:

Se una superficie algebrica ha in un punto 0 la multiplicità $s$, ed il cono $\Gamma$ ivi ad essa tangente possiede una yeneratrice doppia $g$, la quale abbia in 0 raccolte $s+1+t(t \geqslant 0)$ delle sue intersezioni colla superficie, può accadere che $g$ incontri in 0 la prima polare di un punto generico rispetto ad $F s+t$ volte, oppure un numero minore, $s+h(0 \leqslant h<t)$, di volte. Nel primo caso il punto 0 diminuisce la classe della superficie almeno di $s(s-1)^{2}+t+1$ unità, e la diminuisce maggiormente soltanto allorchè $g \grave{e}$ pel cono $\Gamma$ una generatrice cuspidale; nel secondo caso il punto 0 diminuisce la classe di almeno $s(s-1)^{2}+h+2$ unità $\left(^{*}\right)$.

$\mathrm{Ha}$ un interesse particolare il caso in cui 0 è doppio per $F(s=2)$ : la classe di $F$ vien da esso diminuita di più che due unità soltanto quando il cono ivi tangente si spezza in due piani (distinti o no). Se 0 è biplanare, il teorema precedente diviene:

Avendosi una superficie dotata di un punto biplanare 0 , siffatto che la retta $g$ comune ai due piani ivi tangenti abbia $3+t(t \geqslant 0)$ delle sue intersezioni colla superficie raccolte in 0 , questo punto abbassa la classe della superficie precisamente di $t+3$ unità, oppure di almeno $h+4$ unità, secondo che esso assorbe $t+2$, oppure un numero minore, $h+2(0 \leqslant h<t)$, delle intersezioni di g colla prima polare di un punto generico rispetto alla superficie.

Di questo teorema sono immediate conseguenze alcune proprietà che sono state stabilite dal sig. RoHs $\left(^{*}\right)$ ricorrendo a sviluppi in serie: un punto bi-

(*) Di questo teorema non sono noti clie alcuni casi molto particolari (e del resto anche con minor precisione di quella che è nel testo): per $t=0,1$, efr. Ronx, Das Verhalten der Hesse' schen Fläche ecc. (Math. Ann. Bd. XXIII, nota a jag. 89); e per una superficie del $4 .^{\circ}$ ordine con un punto triplo, v. Rons, Ueber die Flächen vierter Ordnung mit dreifachem Punkle (Math. Ann., Bd. XXIV), n. 52,53 e 59, dove l'A. ricorre a sviluppi in serie.

(**) RoHN, Ein Beitrag zur Theorie der biplanaren und uniplanaren Knotenpunkte (Math. Ann., Bd. XXII, pag. 133-134). 
planare può abbassare la classe della superficie (compatibilmente coll' ordine di questa) di quante si vogliano unità, senza che le sezioni fatte nella superficie dai piani in esso tangenti abbiano in quel punto una multiplicità superiore a tre; se la classe vien diminuita di più che tre unità, le sezioni della superficie con ciascuno di quei due piani hanno in generale in quel punto un punto triplo, di cui una delle tangenti è l'intersezione dei due piani tangenti, e non è necessario che intervengano altre particolarità : ecc.

Si deduce altresì :

Perchè un punto biplanare di una superficie algebrica diminuisca la classe di questa precisamente di tre unità, ̀̀ necessario e sufficiente che la retta comune ai due piani ivi tangenti abbia tre, e non più, delle sue intersezioni colla superficie riunite in quel punto.

'Torino, 3 gennaio 1896. 